OPEN ACCESS

Edited by:

Katia Sivieri,

São Paulo State University, Brazil

Reviewed by:

Fabien Magne,

University of Chile, Chile

Carmen Wacher,

National Autonomous University

of Mexico, Mexico

Kiiyukia Matthews Ciira,

Mount Kenya University, Kenya

*Correspondence:

Xue Han

xhan@hit.edu.cn

Shilong Jiang

jiangshilong@feihe.com

Lanwei Zhang

zhanglanwei@ouc.edu.cn

Specialty section:

This article was submitted to

Food Microbiology,

a section of the journal

Frontiers in Microbiology

Received: 04 November 2020

Accepted: 06 July 2021

Published: 13 August 2021

Citation:

Lyu L, Zhou X, Zhang M, Liu L,

Niu H, Zhang J, Chen S, Gong P, Jiang S, Pan J, Li Y, Han X, Cheng D and Zhang L (2021) Delivery Mode Affects Intestinal Microbial Composition and the Development of Intestinal Epithelial Cells.

Front. Microbiol. 12:626144. doi: 10.3389/fmicb.2021.626144

\section{Delivery Mode Affects Intestinal Microbial Composition and the Development of Intestinal Epithelial Cells}

\author{
Linzheng Lyu' ${ }^{1}$, Xiaohong Zhou', Meng Zhang ${ }^{3}$, Li Liu ${ }^{3}$, Haiyue Niu', Jiliang Zhang ${ }^{1}$, \\ Shiwei Chen ${ }^{1}$, Pimin Gong ${ }^{4}$, Shilong Jiang ${ }^{5 *}$, Jiancun Pan ${ }^{5}$, Yuanyuan Li $^{5}$, Xue Han ${ }^{1 *}$, \\ Dayou Cheng ${ }^{1}$ and Lanwei Zhang ${ }^{4 *}$
}

'School of Chemistry and Chemical Engineering, Harbin Institute of Technology, Harbin, China, ${ }^{2}$ Qingdao Central Hospital, Qingdao, China, ${ }^{3}$ The Third Affiliated Hospital of Heilongjiang University of Chinese Medicine, Harbin, China, ${ }^{4}$ College of Food Science and Engineering, Ocean University of China, Qingdao, China, ${ }^{5}$ Heilongjiang Feihe Dairy Co., Ltd., Beijing, China

Background: The infant's intestine contains diverse microbiota, which play an important role in an infant's health.

Objective: This study aimed to analyze the different intestinal microbiota and their function in two delivery modes [vaginal delivery and cesarean section (C-section)] and to investigate the proprieties of bacteria associated with vaginal delivery on the development of intestinal epithelial cells in rat pups.

Materials and Methods: We evaluated the intestinal microbial diversity of the stool samples of 51 infants of subjects who underwent vaginal delivery and C-section by sequencing the V4 regions of the 16S rRNA gene and predicted the function of the microbiotas. The infant stool microbiota in the vaginal delivery group was associated with the digestive system and cell growth and death, whereas that of the C-section group was associated with membrane transport. Then, we isolated the strains based on function prediction.

Results: A total of 95 strains were isolated in the vaginal delivery group. Bifidobacterium bifidum FL-228.1 (FL-228.1) was screened and selected owing to its good surface hydrophobicity, bacterial survivability in the simulated gastrointestinal condition and adhesion ability to the IEC-6 cell line as well as owing to the development of intestinal epithelial cells. Furthermore, in vivo experiments revealed that FL-228.1 exhibited favorable effects on the development of intestinal epithelial cells in rat pups.

Conclusion: The results of this study indicate an apparent difference in the bacterial composition of the stool samples collected from infants of the two delivery modes. By analyzing and screening the bacteria in infant stool samples, we found that one strain, i.e., B bifidum FL-228.1, exhibited favorable effects on the development of intestinal epithelial cells.

Keywords: delivery modes, intestinal microbiota, bacterial screening, infant intestinal epithelial cells, cell proliferation 


\section{INTRODUCTION}

The human intestinal microbiota comprises trillions of microbes and contains millions of functional genes that are mutually beneficial to the host (Friedrich, 2013). After birth, an infant's intestine begins to be colonized by various microorganisms, including Enterobacteria, Enterococci, Staphylococci, Bifidobacteria, and Lactobacilli (Nagpal et al., 2017). These microbiota colonized on the intestine play an important role in infant growth and affect the child's overall health status (Maldonadolobón et al., 2015).

Research has shown that the diversity and composition of the intestinal microbiota in early infants are greatly influenced by external factors (Madan et al., 2016b). Furthermore, many studies have reported that delivery mode is an important factor affecting the composition of the intestinal microbiota throughout the neonatal period and into infancy (Shao et al., 2019). During vaginal delivery, the microorganisms in the vagina are transmitted to the infants vertically. Therefore, Actinobacteria and Bacteroidetes are abundant at the phylum level, whereas Bifidobacterium is the dominant species at the genus level (Lundgren et al., 2018). Compared with vaginal delivery infants, the intestinal microbiota of cesarean section (C-section) infants predominantly originate from the mother's skin as well as from microorganisms in the environment. Therefore, the relative abundance of Firmicutes and a few microbes from the skin, such as Staphylococcus and Clostridium, are higher than that of other bacteria (Jakobsson et al., 2014).

Studies in infants have indicated that C-section infants tend to have lower number of anaerobes (e.g., Bacteroidetes) and a less diverse microbiota than vaginal delivery infants (Chong et al., 2018). Further, the composition of microbiota in vaginal delivery infants is relatively more stable than that of C-section infants until 2 months of life (Reyman et al., 2019). Analysis of the intestinal microbiota of infants has revealed that there are differences in the composition of the intestinal microbiota of C-section and vaginal delivery infants (Shao et al., 2019). Interestingly, although there are apparent differences in stool microbiological composition of C-section and vaginal delivery infants after birth, a study has shown that C-section subjects gradually progress toward harboring microbiota reassembling natural birth infants by week 8 of life, which is maintained till week 24 (Hill et al., 2017).

Previous studies have compared the bacterial composition of stool samples of C-section and vaginal delivery infants (Madan et al., 2016b). Epidemiological evidence has revealed that C-section affects the initialization of intestinal microbiota colonization in infants, which in turn affects the formation and maturation of the intestinal immune system (Planer et al., 2016). A study by Salam (Salam et al., 2006) reported that C-section damages intestinal immunity by increasing the risk of gastrointestinal infections and allergies. However, to the best of our knowledge, no study has screened and isolated bacteria based on these two delivery modes for functional exploration. As a result, this study aimed to analyze the different intestinal microbiota and their function in the two delivery modes as well as to investigate the proprieties of bacteria associated with vaginal delivery on the development of intestinal epithelial cells in rat pups.

\section{MATERIALS AND METHODS}

\section{Participants}

Fifty-one healthy families were recruited from the Third Affiliated Hospital of Heilongjiang University of Chinese Medicine (China) and Harbin Children's Hospital (Harbin, China). The Institutional Review Board of Harbin Children's Hospital approved this study; written informed consent was obtained from all the mothers. Prior to sampling, we surveyed the mothers via a questionnaire to collect clinical data. Infant stool samples were collected at the hospital or during a home visit in a period of 30 days. They were promptly transported to the laboratory and stored at $-80^{\circ} \mathrm{C}$ until further analysis (Bäckhed et al., 2015).

\section{Isolation of Different Strains}

One gram of the stool sample was diluted with $9 \mathrm{~mL}$ of phosphate-buffered saline (PBS buffer). The semi-selective medium method was used to screen the strains, as shown in Table 1. The plates were anaerobically incubated at $37^{\circ} \mathrm{C}$ for $72 \mathrm{~h}$ and then left in the air for $4 \mathrm{~h}$. Single, blue colonies were isolated and subcultured for further purification and experiments. Each selected strain was subjected to microscopic examination and gram staining. The gram-positive strains were selected for further research (Riaz Rajoka et al., 2017).

\section{Surface Hydrophobicity}

The surface hydrophobicity of the selected strains was determined using a previously described method with some modifications ( $\mathrm{Xu}$ et al., 2018). Briefly, the bacteria were incubated at $37^{\circ} \mathrm{C}$ for $72 \mathrm{~h}$, centrifuged for $3 \mathrm{~min}$ at 6000 $\mathrm{r} / \mathrm{min}$ and then resuspended in $3 \mathrm{~mL}$ of sterile PBS buffer. The absorbance of the suspension was measured at $600 \mathrm{~nm}\left(\mathrm{~A}_{0}\right)$. Then, the suspension was mixed with $1 \mathrm{~mL}$ of ethyl acetate and placed at room temperature for $10 \mathrm{~min}$. The two-phase system was mixed via swirl oscillation for $2 \mathrm{~min}$. Then, the two-phase system was reconstructed by standing for $20 \mathrm{~min}$. The aqueous phase was carefully removed, and the absorbance was measured at $600 \mathrm{~nm}\left(\mathrm{~A}_{1}\right)$. The reference strain Lactobacillus rhamnosus LGG (ATCC 53103) was used as a control. Surface hydrophobicity was calculated according to the following formula (Pan et al., 2006):

$\mathrm{H} \%=\left(1-\mathrm{A}_{1} / \mathrm{A}_{0}\right) \times 100$

TABLE 1 | Semi-selective medium.

\begin{tabular}{lll}
\hline $\begin{array}{l}\text { Putatively } \\
\text { common } \\
\text { strains }\end{array}$ & Additive & $\begin{array}{l}\text { Semi-selective medium (Martín } \\
\text { et al., 2009; Lau et al., 2016) }\end{array}$ \\
\hline Lactobacillus & $\begin{array}{l}\text { Nalidixic acid } \\
\text { and X-gal }\end{array}$ & MRS-Cys/LBS-Cys \\
Bifidobacterium & $\begin{array}{l}\text { Nalidixic acid } \\
\text { and X-gal }\end{array}$ & MRS-Cys/LBS-Cys \\
\end{tabular}




\section{Bacterial Survivability in a Simulated Gastrointestinal Condition}

The isolates were incubated in a simulated gastric fluid of PBS buffer supplemented with pepsin $(0.5 \%)$ and adjusted to $\mathrm{pH}$ 2.5 with $0.1 \mathrm{M}$ hydrochloric acid. After incubation at $37^{\circ} \mathrm{C}$ for $1.5 \mathrm{~h}$, the viable isolates were evaluated via plate count on MRS agar. The simulated intestinal juice tolerance of the isolates that survived for $2 \mathrm{~h}$ in the simulated intestinal juice was determined using PBS buffer containing 0.3\% (w/v) bile salt and trypsin $(1 \mathrm{mg} / \mathrm{mL})$; the $\mathrm{pH}$ was adjusted to 8.0 . Following a 2$\mathrm{h}$ incubation at $37^{\circ} \mathrm{C}$, the simulated intestinal juice was assessed by measuring the survived isolates using MRS agar medium (Xu et al., 2018). The reference strain Lactobacillus rhamnosus LGG (ATCC 53103) was used as a control.

\section{Bacterial Adhesion Ability to the IEC-6 Cell Line}

IEC-6 (National Collection of Authenticated Cell Cultures) were incubated in RPMI-1640 medium supplemented with 10\% heatinactivated fetal bovine serum. For adhesion assays, IEC- 6 cells were seeded into 24 -well tissue culture plates at a concentration of $2.5 \times 10^{5}$ cells/well and incubated at $37^{\circ} \mathrm{C}$ for $24 \mathrm{~h}$ in $5 \% \mathrm{CO}_{2}$ and $95 \%$ air. Then, the bacteria were added into the corresponding wells. Following 4-h incubation, IEC-6 cells were washed with PBS buffer to remove the bacterial suspensions, followed by lysis of non-adherent bacteria with $0.1 \%$ Triton X-100 solution. The adherent bacteria were serially diluted and spread on MRS agar plates. The plates were incubated in an anaerobic environment at $37^{\circ} \mathrm{C}$ for $72 \mathrm{~h}$ to calculate the number of adherent bacteria. The reference strain Lactobacillus rhamnosus LGG (ATCC 53103) was used as a control. The adhesion percentage of the bacteria was calculated according to the following formula ( $\mathrm{Oh}$ and Jung, 2015):

Adhesion ability rate $(\%)=$ [adhered cell number (log $\mathrm{CFU} / \mathrm{mL}) /$ initial cell number $(\log \mathrm{CFU} / \mathrm{mL})] \times 100 \%$

\section{Development of Intestinal Epithelial Cells in vitro}

A concentration of $2.5 \times 10^{5}$ cells $/ \mathrm{mL}$ was inoculated into a 24 well plate; the culture medium was changed after overnight culturing at $37^{\circ} \mathrm{C}$. Then, bacteria were added into the corresponding wells at the ratio of 1:100. The bacteria and IEC-6 cells were cultured together for $4 \mathrm{~h}$, followed by washing IEC6 cells with PBS buffer. The blood cell counting plate was used to count the number of IEC- 6 cells in each well. The reference

TABLE 2 | Intervention treatment.

\begin{tabular}{ll}
\hline Groups & Intervention treatment \\
\hline Blank control group & Normal lactation and intragastric administration of PBS \\
Positive control group & $\begin{array}{l}\text { Normal lactation and intragastric administration of LGG } \\
\text { PBS suspension }\left(10^{9} \mathrm{CFU} / \mathrm{mL}\right)\end{array}$ \\
Experimental group & Normal lactation and intragastric administration of the \\
& FL-228.1 strain in a PBS suspension $\left(10^{9} \mathrm{CFU} / \mathrm{mL}\right)$
\end{tabular}

strain Lactobacillus rhamnosus LGG (ATCC 53103) was used as a control. The experiment was repeated three times.

\section{Identification of the Different Strains}

The 16S rRNA gene sequencing method was used to identify the isolated strains. Briefly, genomic DNA was extracted using the E.Z.N.A. bacterial DNA kit (Omega Bio-Tek, Norcross, GA). The 16S rRNA gene was amplified using the universal primers 27F (5'-AGAGTTTGATCCTGGCTCAG-3') and 1492R (5'-TACGGTTACCTTGTTACGACTT-3') (Meng et al., 2018). The amplicons were sequenced by Sangon Biotech Co., Ltd. (Shanghai, China). Sequence similarity analysis was performed by comparing the sequences with those in GenBank via the BLAST search program of the National Centre for Biotechnology Information. ${ }^{1}$

\section{Establishment of the Intrauterine Growth Retardation (IUGR) Animal Model}

Pregnant female Sprague Dawley rats were provided by Charles River Laboratory Animal Technology Co., Ltd. (Beijing, China). Pregnant rats were fed separately. The IUGR animal model was established via maternal nutritional restriction. Pregnant rats were randomly divided into two groups: Normal diet group and food restriction group. The rats in the normal diet group were allowed ad libitum access to food and water. From the 3rd day of pregnancy to the day of delivery, the rats in food restriction group were provided with $50 \%$ of food. Both groups received adequate food and water on the day of delivery. Compared with normal neonatal rats, IUGR neonatal rats were successfully modeled when the weight of neonatal rats was less than two standard deviations in the food restriction group. Following successful

${ }^{1}$ http://www.ncbi.nlm.nih.gov

TABLE 3 | Basic information of participants.

\begin{tabular}{|c|c|c|c|c|}
\hline \multirow[t]{3}{*}{ Characteristics } & \multicolumn{4}{|c|}{ Delivery modes } \\
\hline & \multicolumn{2}{|c|}{ C-section } & \multicolumn{2}{|c|}{ Vaginal } \\
\hline & $\begin{array}{l}\text { Median } \\
\text { or No. }\end{array}$ & $\begin{array}{l}\text { Interquartile } \\
\text { ranges or } \\
\text { percentage }\end{array}$ & $\begin{array}{l}\text { Median or } \\
\text { No. }\end{array}$ & $\begin{array}{l}\text { Interquartile } \\
\text { ranges or } \\
\text { percentage }\end{array}$ \\
\hline Mother's age (y) & 31 & $22-38$ & 29 & $23-35$ \\
\hline Father's age (y) & 33 & $25-43$ & 29 & $24-37$ \\
\hline $\begin{array}{l}\text { Mother's pregnancy } \\
\text { weight }(\mathrm{kg})\end{array}$ & 74.2 & $57-96$ & 69.75 & $60.6-92.5$ \\
\hline Gestation age (d) & 277 & $266-284$ & 279 & $266-288$ \\
\hline Birth weight (g) & 3725 & $3,000-4,800$ & 3400 & $2,700-3,900$ \\
\hline Birth length (cm) & 52 & $49-55.6$ & 50 & $49-51$ \\
\hline \multicolumn{5}{|l|}{ Infant sex } \\
\hline Male & 18 & $69.23 \%$ & 14 & $56.00 \%$ \\
\hline Female & 8 & $30.77 \%$ & 11 & $44.00 \%$ \\
\hline \multicolumn{5}{|l|}{ Feeding patterns } \\
\hline $\begin{array}{l}\text { Exclusive } \\
\text { breastfeeding }\end{array}$ & 16 & $61.54 \%$ & 12 & $48.00 \%$ \\
\hline Mixed feeding & 10 & $38.46 \%$ & 13 & $52.00 \%$ \\
\hline
\end{tabular}



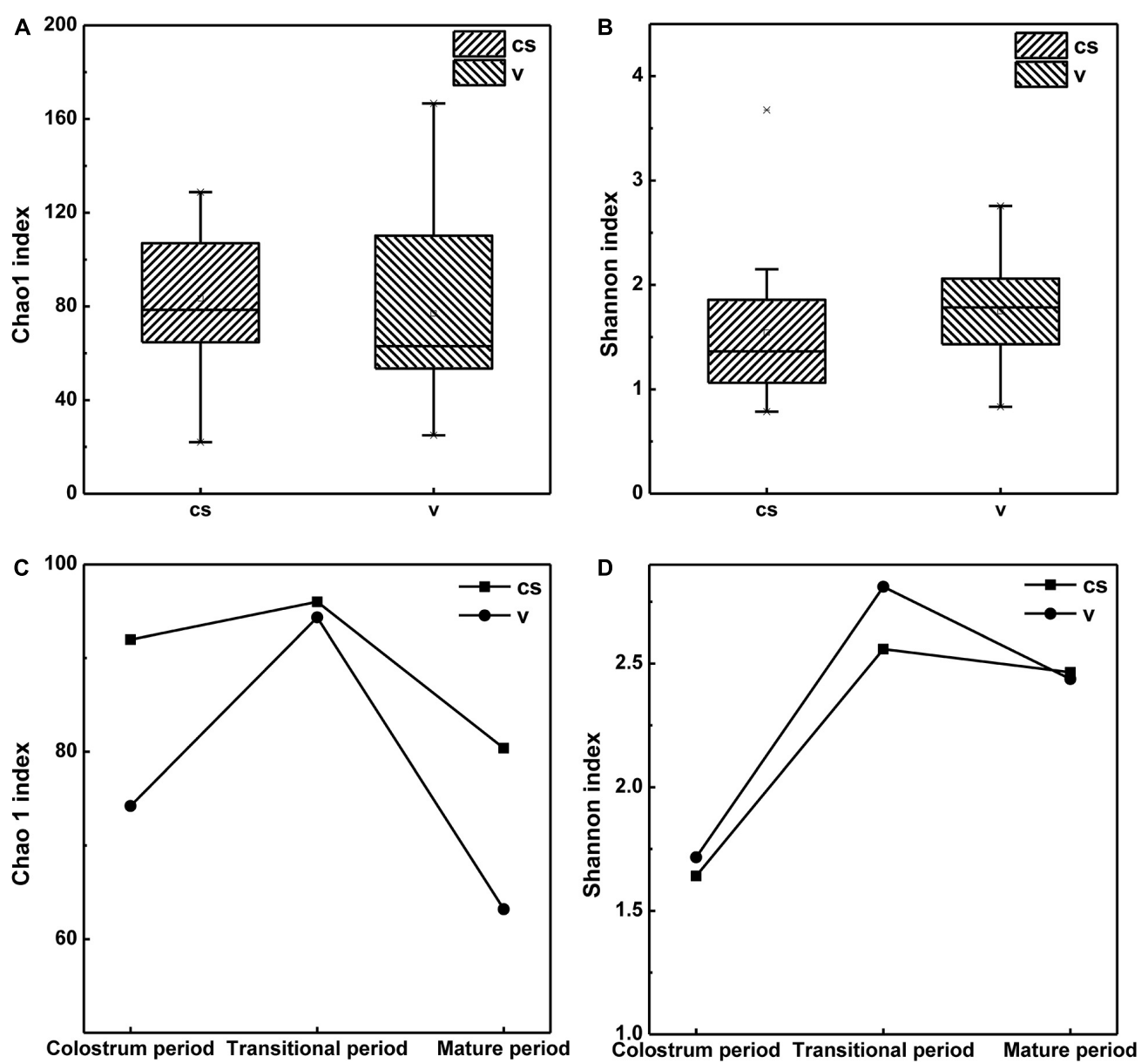

FIGURE 1 | Alpha diversity of the stool microbiota of infant born via different delivery modes. (A) The Chao1 index of infant stool. (B) The Shannon index of infant stool. (C) Changes in Chao1 index in the stool samples of infants in different stages. (D) Changes in Shannon index in the stool samples of infants in different stages.
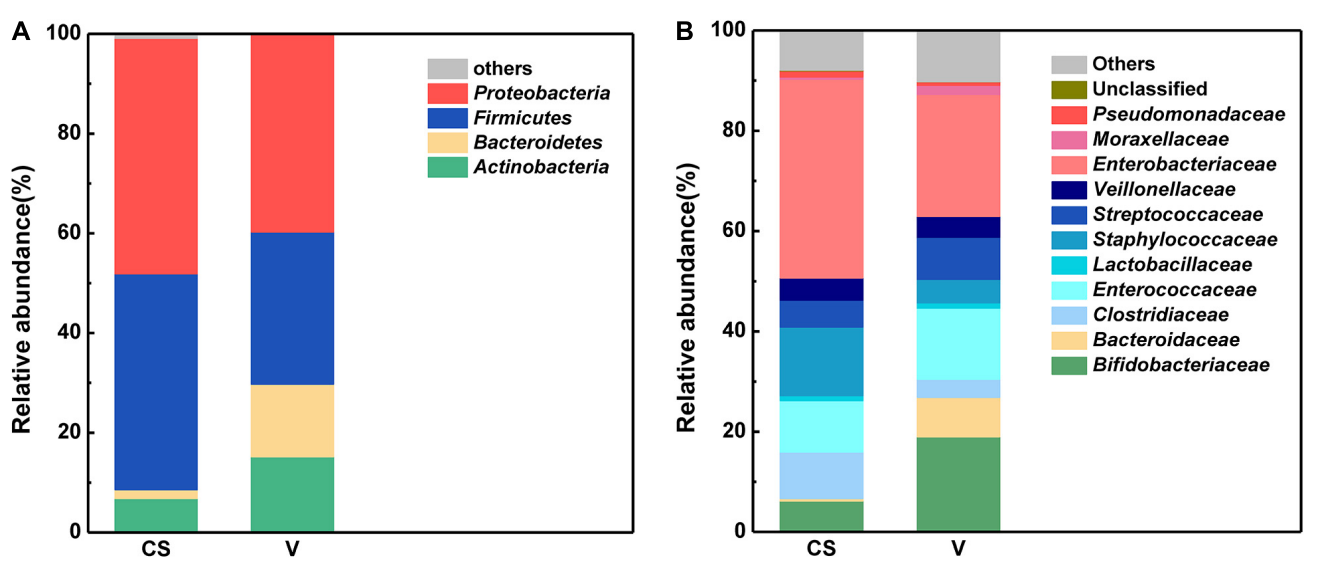

FIGURE 2 | Characteristics of the infant stool microbiota between the two different delivery modes at the phylum and family levels. (A) Phylum level characteristics of infant stool microbiota between the two different delivery modes. (B) Family level characteristics of infant stool microbiota between the two different delivery modes.

modeling, the models were grouped into the following groups, as shown in Table 2: Blank control group, positive control group and experimental group. Neonatal rats were naturally fed for
3 days. Then, the intervention experiment was conducted. The bacteria were supplemented to pups via oral gavage using a special syringe ( $0.6 \mathrm{~mm}$ diameter). Rat pups were anaesthetized 

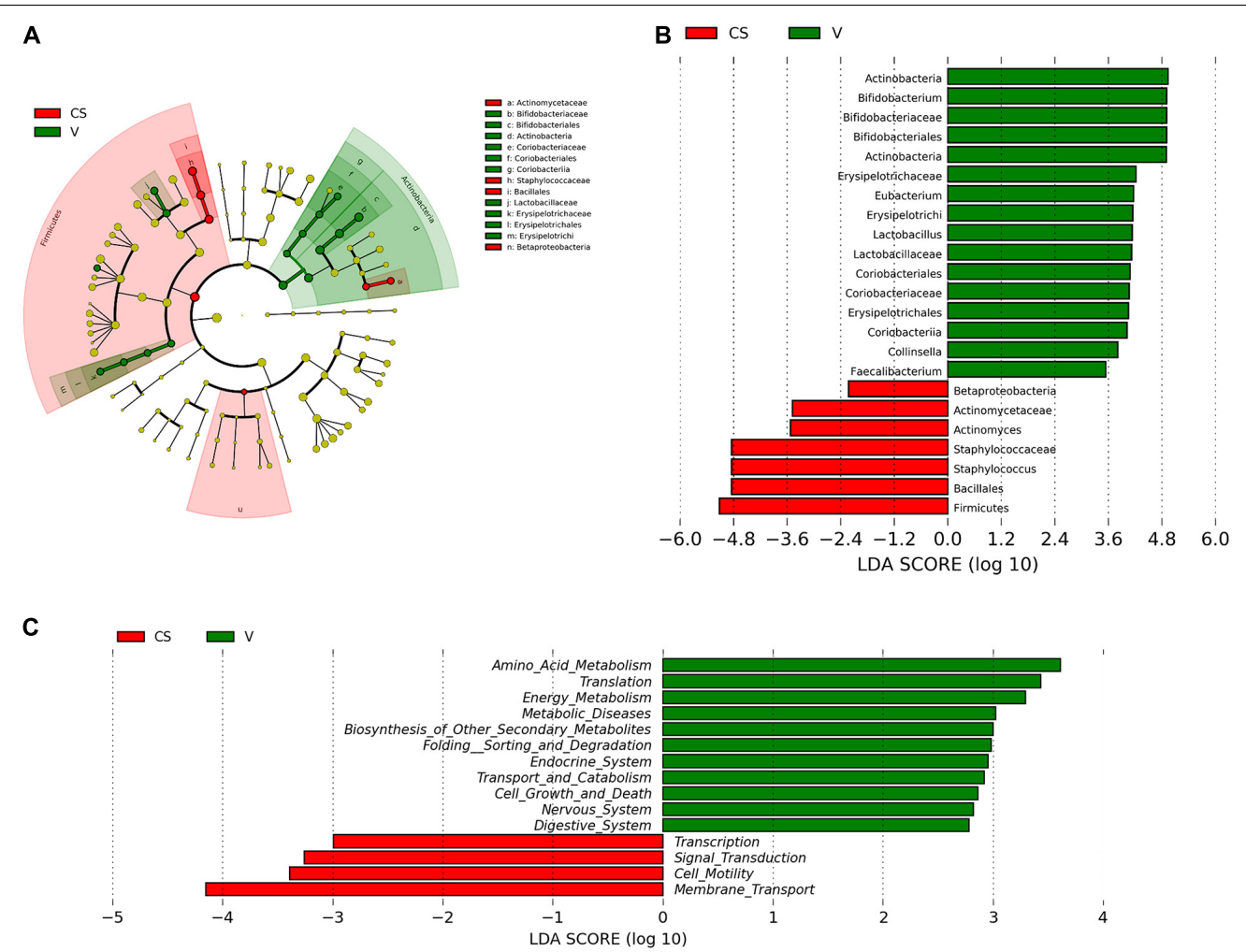

FIGURE 3 | Comparison of the different microbiota in the stool samples of infants born via different delivery modes. (A) LEfSe analysis of the different microbiota in stool samples of vaginal delivery and C-section infants. Microbial communities in the samples of the vaginal delivery infants via their LDA value statistics are shown in green; C-section samples with a negative LDA score are shown in red. LDA value is absolute. (B) Cladogram derived from LEfSe analysis of the metagenomic sequences from the stool samples of C-section and vaginal delivery infants. Green areas indicate a significant effect on the microbial groups in the vaginal delivery group. Red areas indicate a significant effect on the microbial groups in the C-section group. LDA values were obtained via linear regression analysis. (C) Analysis of KEGG differential pathways via LEfSe.

with isoflurane and sacrificed via cervical dislocation on the 10th day (6 pups/group) (Ding et al., 2016).

\section{Intestinal Morphological Characteristics of Rat Pups}

The intestinal morphology of rat pups was assessed via the villus length to determine whether the intestinal structure was affected by bacterial supplementation using the hematoxylineosin -staining. Following dehydration, the fixed intestinal villus tissues were embedded in paraffin, sliced $(5 \mu \mathrm{m})$, patched in warm water at $42^{\circ} \mathrm{C}$ and copied at $42^{\circ} \mathrm{C}$ for $8 \mathrm{~h}$. The hematoxylineosin-stained paraffin sections were observed under a Zeiss Axio Imager microscope (Désir-Vigné et al., 2018).

\section{Effect of Strains on Intestinal Cell Development}

The small and large intestinal tissues of the nuclear development marker Ki-67 were collected via immunostaining on the 10th day to detect the contribution of bacteria toward the changes in intestinal structures. The immunohistochemical method was used to detect the expression of the cell development activity indicator in the intestinal mucosa. Parallel tissue slices of the cells were taken. Rabbit anti-Ki-67 monoclonal antibody was incubated in a wet box at room temperature. HRP-labeled antirabbit IgG was incubated at room temperature for $1 \mathrm{~h}$. The positive result of DAB staining was brown at the antigen location (Bhinder et al., 2017).

\section{Illumina Sequencing and Statistical Analyses}

Total genomic DNA was extracted from infant stool samples using the E.Z.N.A. bacterial DNA kit (Omega Bio-Tek, Norcross, GA) following the manufacturer's instructions. The V4 region of the 16S rRNA gene was sequenced on the HiSeq 2500 sequencer, which was performed by BGI Tech Solutions Co., Ltd. (Shenzhen, China). The microbiome data were then analyzed using the Mothur software (V1.31.2). All samples were analyzed at the operational taxonomic unit (OTU) level. An OTU was defined as a group of bacteria with more than $97 \%$ similarity. Differences in OTUs were analyzed in every sample via $\alpha$-diversity (chao1 and Shannon indices) using the $\mathrm{R}$ package. The bacterial diversity of infant stool samples was analyzed using the $\mathrm{R}$ package (ade4). Community composition analysis between the groups was performed using distance matrices (Adonis, $\mathrm{R}$ vegan package) (Pragman et al., 2018). All linear discriminant analysis effect size (LEfSe) taxonomic information was detected using the 
Metaphlan 2 program, with the cladogram derived from LEfSe analysis (Liu et al., 2016). The Illumina sequences were submitted to the NCBI Sequence Read Archive database with an accession number of PRJNA683130. The 16S rRNA sequence of FL-228.1 was submitted to the GeneBank database with an accession number of MT071594.

All experiment results were analyzed with SPSS software (version 22). $P$-values less than 0.05 were considered statistically significant.

\section{RESULTS}

\section{Questionnaire and Subject Information}

Fifty-one healthy infants were recruited; their basic information is summarized in Table 3. Among the infants recruited, there were 32 boys and 19 girls. In total, 25 (49\%) infants were born via vaginal delivery and 26 (51\%) were born via C-section.

\section{Biodiversity of the Intestinal Microbiota of Infant Born via Different Delivery Modes}

Within-sample (alpha) diversity of the infant stool samples was assessed using two indices, namely, the Shannon and Chaol indices. As shown in Figure 1A, for all samples, the microbiota of C-section infants exhibited higher Chaol index than those of vaginal delivery infants; however, the difference was not significant. This indicated that the infants in the vaginal delivery group had a higher number of bacterial species than the infants in the C-section group. Meanwhile, the microbiota in the stool samples of C-section infants showed a non-significantly lower Shannon index than those in stool samples of vaginal delivery infants (Supplementary Table 1). This result suggested that vaginal delivery had higher evenness in infant stool (Figure 1B).

All samples were grouped according to infants' age: 1-7 days (colostrum period), 8-14 days (transitional period) and 1530 days (mature period). The results also indicated that after birth, the bacterial species in the infant stool had increased from the 1st day to the 14th day after birth and then declined until the 30th day. Shannon index also showed a similar trend, indicating that the evenness of the intestinal microbiota was richest during the transitional period and then decreased during the maturation period. Both Chaol and Shannon indices showed the highest values during the transitional period, whether in the stool samples of C-section infants or in those of vaginal delivery infants (Figures 1C,D). This meant that there were more species and bacterial homogeneity in the transitional period than in the other periods both in the $\mathrm{C}$-section and vaginal delivery modes.

\section{Bacterial Components in the Infant's Intestine Between the Two Delivery Modes}

The relative abundance of the different bacteria in the infants born via the two delivery modes is depicted in Figure 2 . Proteobacteria and Firmicutes were the dominant phyla observed in these two delivery modes (Figure 2A). Further, the number of
Proteobacteria and Firmicutes was higher in the C-section group than in the vaginal delivery group. In contrast, Bacteroidetes (Bacteroidaceae) and Actinobacteria (Bifidobacteriaceae) occupied only a small percentage compared with the above two phyla. At the family level (Figure 2B), Enterobacteriaceae exhibited the highest relative abundance in the C-section group, followed by Staphylococcaceae and Lactobacillaceae. In contrast, Enterobacteriaceae, Bifidobacteriaceae, and Lactobacillaceae were dominant in the vaginal delivery group, and other families only occupied a small percentage.

LEfSe and cladistics analyses were performed to investigate the differences in the community composition between the two different delivery modes. At the phylum level, Actinobacteria and Firmicutes were dominant in both groups. At the family level, there were five different families, with enrichment of Bifidobacteriaceae, Erysipelotrichaceae, Lactobacillaceae, Coribacteriaceae, and Coribacteriaceae in the vaginal delivery group and that of Actinomycetaceae and Staphylococcaceae in the C-section group (Figures 3A,B). There were seven different genera between the two groups. Bifidobacterium, Eubacterium, Lactobacillus, Collinsella, and Faecalibacterium exhibited a relatively high abundance in the vaginal delivery group, whereas Actinomyces and Staphylococcus were more abundant in the C-section group (Figures 3A,B).

\section{Function Prediction of Infant's Intestinal Microbiota Between the Two Delivery Modes}

LEfSe analysis was performed to analyze the different functions between the two delivery modes. The abscissa is the log value of the value obtained via linear regression analysis (LDA), as depicted in Figure 3C. According to function prediction of the microbiota (Figure 3C), it could be concluded that the infant stool microbiota in the $\mathrm{C}$-section group was related to membrane transport, cell motility, signal transduction and transcription. In contrast, the infant stool microbiota in the vaginal delivery group was associated with the digestive system.

\section{Strain Isolation}

Semi-selective medium was used to isolate the target strains from 25 vaginal-delivered infant stool samples. A total of 95 strains were isolated from 25 vaginal-delivered infant stool samples. Among them, 40 putative Lactobacillus and Bifidobacterium strains were screened via microscopic examination and colony morphology analysis.

\section{Surface Hydrophobicity of the Isolates}

The hydrophobicity method was used to screen the strains, as shown in Figure 4A. The surface hydrophobicity of the 40 putatively differential strains ranged from 28.31 to $63.47 \%$, as shown in Figure 4A. Among them, 15 strains were screened because they had higher hydrophobicity percentages than the reference strain LGG (52.26\%); in particular, the strain named FL-228.1 exhibited the highest hydrophobicity (63.47\%). On average, Lactobacillus (51.62\%) showed better hydrophobicity than Bifidobacterium (47.61\%). 

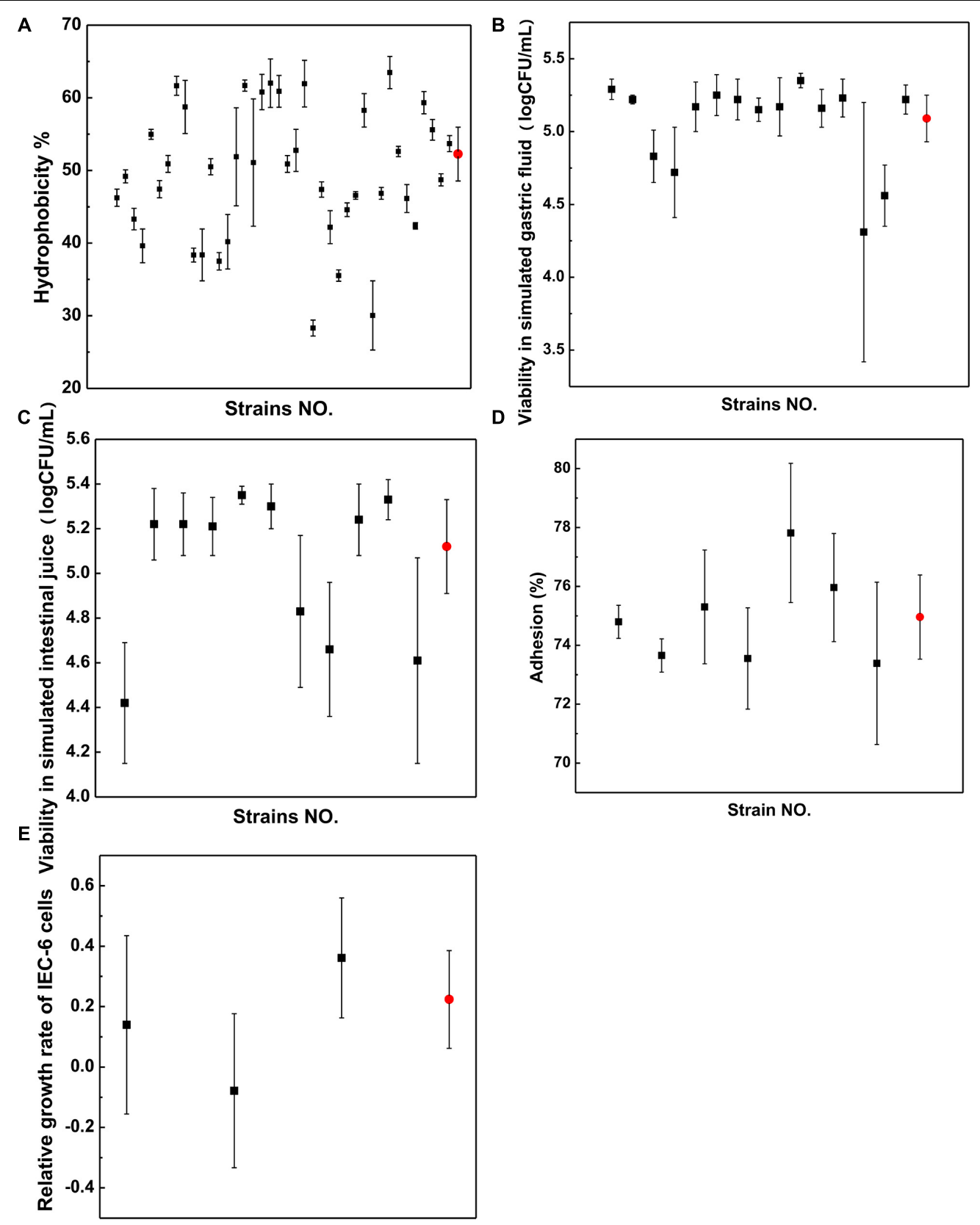

Strains NO.

FIGURE 4 | Properties of strains. (A) Surface hydrophobicity. (B) Viability in simulated gastric fluid. (C) Viability in simulated intestinal juice. (D) Adhesion ability. (E) Relative growth rate. Black dots represent strains from infant stool samples and red dots represent those from LGG.

\section{Gastrointestinal Tract Viability of the Isolates}

The survival rates of 11 strains showed higher resistance to acidic conditions than LGG and were screened following exposure to simulated gastric fluid from the 15 strains, as shown in Figure 4B. The survival rates of seven strains were screened following exposure to simulated intestinal juice from 11 strains, as shown in Figure 4C. The results indicated that compared with LGG, the seven strains showed higher resistance to the simulated intestinal juice condition. The isolate FL-228.1 showed the highest resistance. A total of seven strains were screened for surface hydrophobicity and gastrointestinal tolerance indicator.

\section{Adhesion Ability of the Different Strains to IEC-6 Cells}

Through gastrointestinal fluid simulation and hydrophobicity experiments, seven bacterial strains were screened for further cell adhesion tests. Compared with LGG, three isolates from the seven 


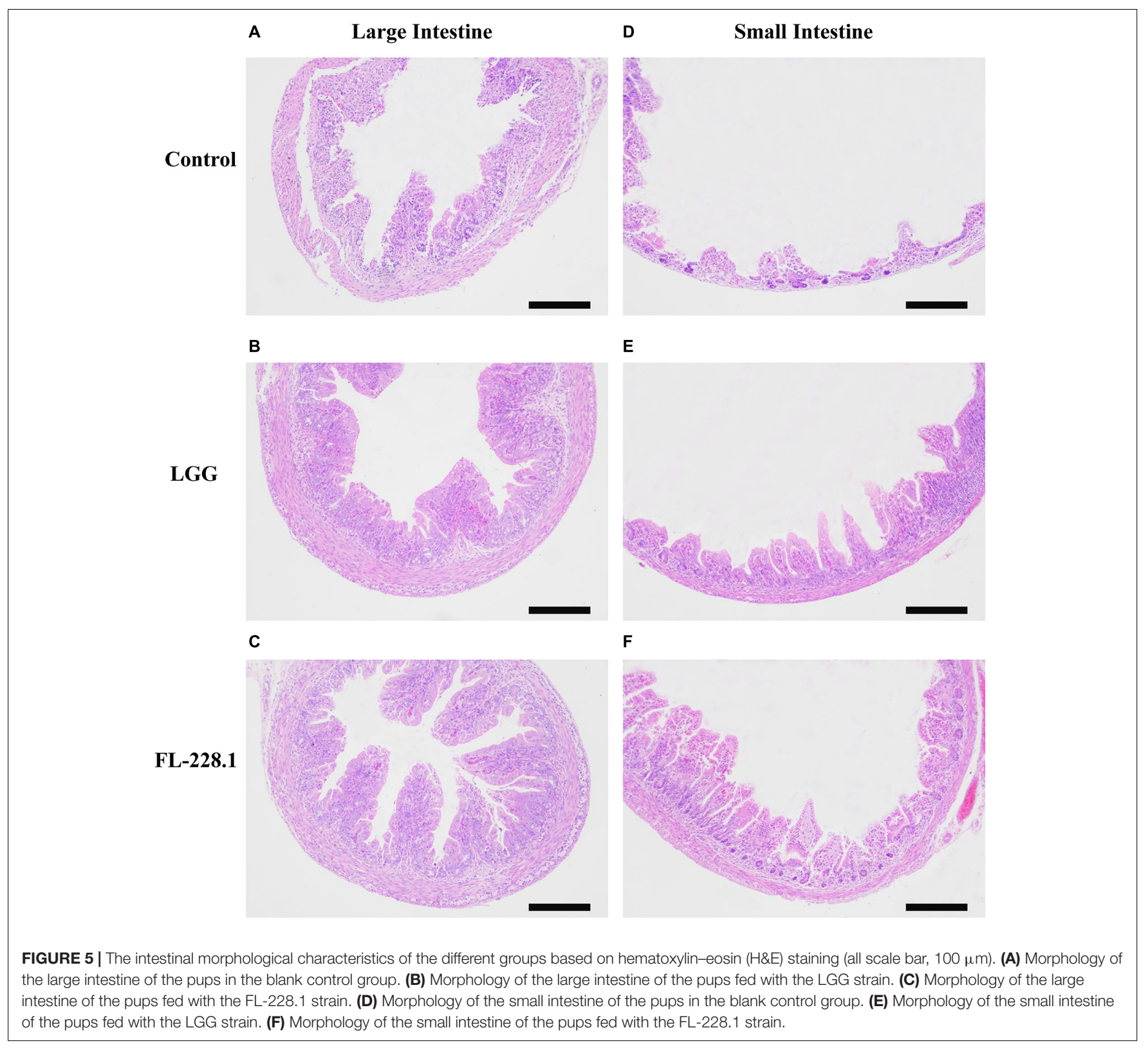

strains, namely, FL-215, FL-216.9 and FL-228.1, showed higher adhesion abilities to IEC- 6 cells. Further, among the three isolates, FL-228.1 showed the strongest adhesion ability to IEC-6 cells, as shown in Figure 4D. The specific results of the adhesion ability of the different strains are shown in Supplementary Table 2.

\section{Effect of the Isolates on the Development of Intestinal Epithelial Cells in vitro}

Through cell adhesion tests, three bacterial strains were screened for promoting the development of intestinal epithelial cells. The in vitro study revealed that FL-215 and FL228.1 promoted the development of intestinal epithelial cells and that FL-216.9 could not function in promoting the development of intestinal epithelial cells. Further, FL-228.1 showed improvements compared with the LGG group in terms of the relative growth rate of IEC-6 cells (Figure 4E).

\section{Effect of $B$. bifidum FL-228.1 on the Intestinal Morphological Characteristics of Rat Pups}

The morphological characteristics of the different parts of the rat pup's intestine after intervention with the FL-228.1 strain for 10 days are depicted in Figure 5. Compared with the blank control group and LGG, the pups fed with FL-228.1 exhibited longer large intestine villi (Figures 5A-C). Furthermore, FL228.1 exhibited longer small intestine villi in the offspring compared with blank control group and LGG (Figures 5D-F). 


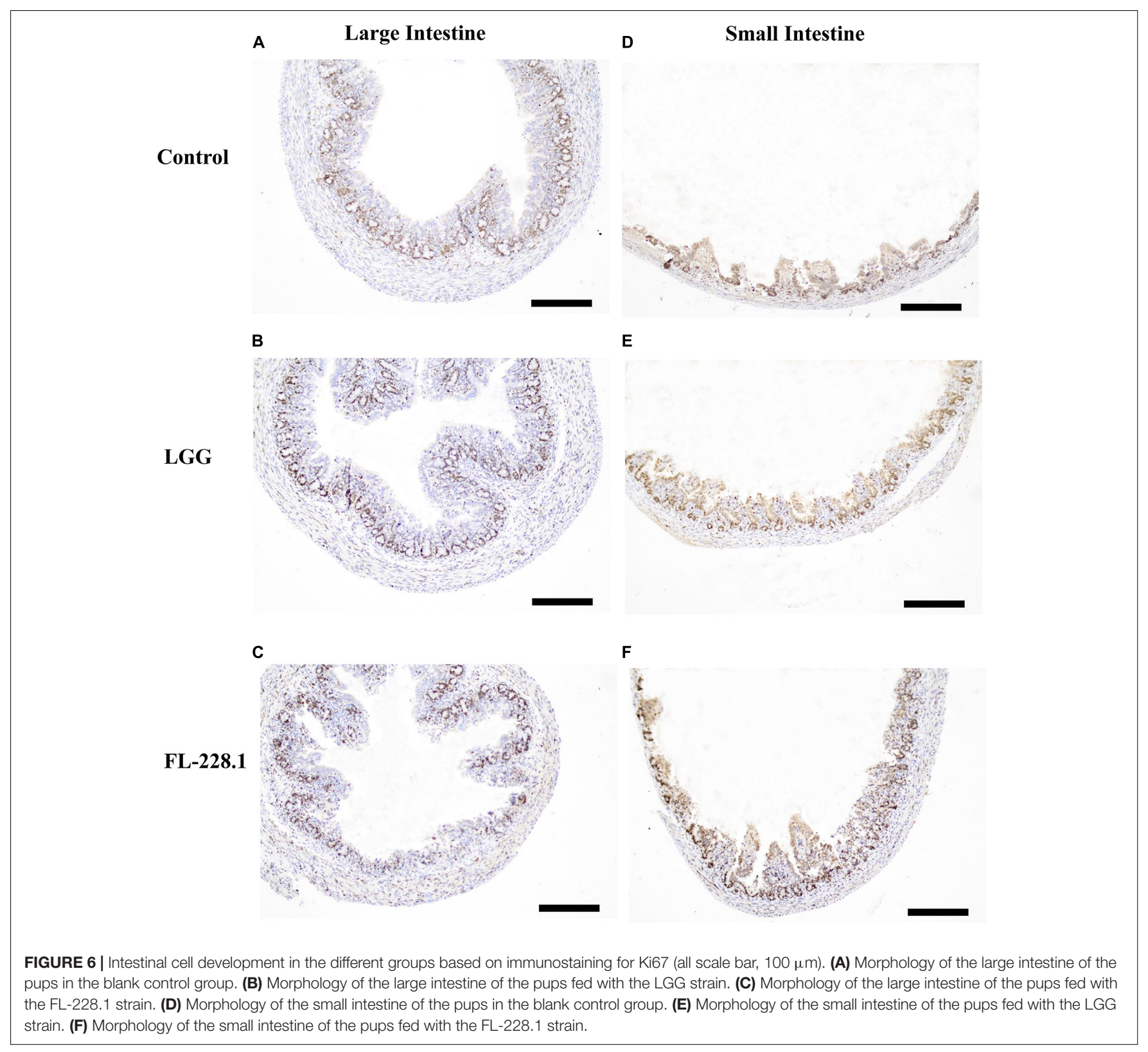

Therefore, FL-228.1 promoted the development of small and large intestinal villi in the offspring.

\section{Effect of B. bifidum FL-228.1 on Intestinal Cell Development in the Rat Pups}

Following intervention with the FL-228.1 strain for 10 days, intestinal cell development in the rat pups is depicted in Figures 6, 7. Compared with the blank and LGG groups, the mean densities of the large intestine and small intestine of the rats fed with FL-228.1 were the highest. Furthermore, for the large intestine, the mean density of the FL-228.1-fed pups was significantly higher than that of LGG-fed or blank groups $(P<0.05)$. The mean density of the LGG-fed pups was significantly higher than that of the control group $(P<0.05)$
(Figure 7A). Moreover, in the small intestine, the mean density of the FL-228.1-fed pups was significantly higher than that of LGGfed and blank groups $(P<0.05)$ (Figure 7B). Therefore, FL-228.1 can promote intestinal cell development in the small and large intestines of the offspring.

\section{DISCUSSION}

\section{Effect of Different Delivery Modes on the Composition of Infant Intestinal Microbiota}

The 16S rRNA gene Illumina HiSeq platform was used to analyze stool samples from healthy infants. Although our study was 

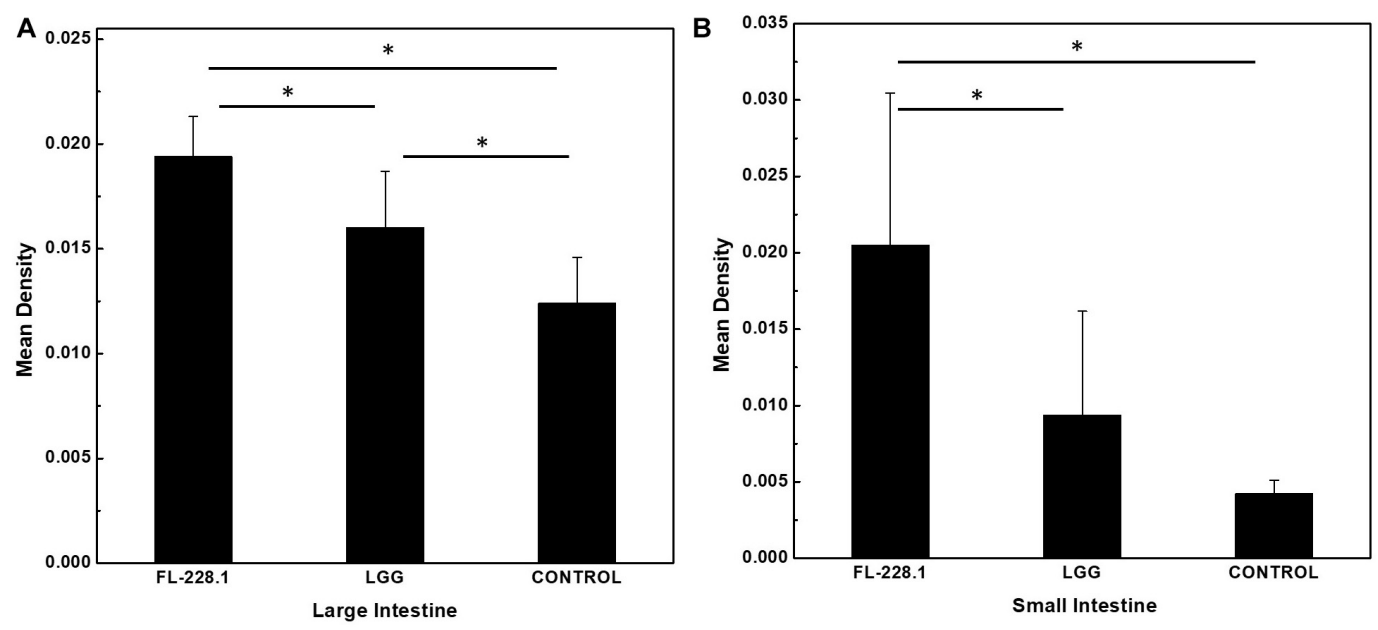

FIGURE 7 | The mean density of the small and large intestines of the different groups. (A) The mean density of the large intestine of the different groups. (B) The mean density of the small intestine of the different groups. ${ }^{*} P<0.05$.

limited by sequencing depth and length, to our knowledge, our study was the first to investigate the microbial diversity of infant stool samples from two delivery methods from subjects living in northeast China.

We observed some associations among stool microbiota, community composition and modes of delivery (Figure 2). At the phylum level, the most abundant bacterial phyla were Firmicutes and Proteobacteria in these two delivery modes. This result is consistent with that of a recent investigation in another cohort (Wampach et al., 2018). Moreover, the most apparent differences at the family level were the relative abundances of Bifidobacteriaceae, which were dominant in the vaginal delivery group. This result was generally consistent with that of previous studies, which found a greater relative abundance of Bifidobacteriaceae in the stool samples of vaginal delivery infants (Shao et al., 2019). In contrast, the relative abundance of Clostridiaceae and Staphylococcaceae was higher in the stool samples of C-section infants presented by mother's skin and the hospital environment (Madan et al., 2016a; Tamburini et al., 2016; Shi et al., 2018). Moreover, samples from vaginal delivery infants were enriched with Bacteroidaceae; this result is consistent with that of a recent investigation in another cohort (Wampach et al., 2018).

There were apparent differences in infant intestinal microbiota in the two different delivery modes. According to the results of biodiversity analysis (Figures $3 \mathbf{A}, \mathbf{B}$ ), we found that Bifidobacterium and Lactobacillus were the different intestinal microbiota in the vaginal delivery and C-section groups. Meanwhile, according to the results of LEfSe analysis and function prediction, it was speculated that different bacterial groups lead to different functions. The vaginal delivery group was associated with the digestive system and cell growth and death. Function prediction could provide guidance for subsequent bacterial isolation and functional verification. Therefore, we analyzed the potential efficacies of these two genera, which are anaerobic and gram-positive. Meanwhile, many studies have confirmed that Bifidobacterium and Lactobacillus have certain effects on intestinal development (Mi et al., 2017; O'Connell Motherway et al., 2019; Zhou et al., 2020). Therefore, we analyzed only anaerobic and gram-positive bacterial strains and used Bifidobacterium and Lactobacillus as our target strains.

\section{Bacterial Strains Promote Intestinal Development}

Bifidobacterium and Lactobacillus had important effects on intestinal development (Blanton et al., 2016; Mi et al., 2017; O'Connell Motherway et al., 2019; Zhou et al., 2020). The tight adhesion (Tad) pili of Bifidobacterium is an important colonizing factor that promotes the development of intestinal epithelial cells (O'Connell Motherway et al., 2019). It was confirmed that the development of intestinal epithelial cells significantly increased 5 days after Bifidobacterium ingestion in mice. Furthermore, the Tad pili of Bifidobacterium promoted the intestinal mucosal growth of neonates by producing specific extracellular protein scaffolds, thereby promoting intestinal maturation of early neonates (O'Connell Motherway et al., 2019). This result is consistent with our results as FL-228.1 exhibited greater ability to develop in the intestine than the LGG and blank control group. Another study demonstrated that Bif. longum can enhance intestinal development and mucosal repair, promote lysozyme production and ameliorate dysbiosis of the microbiota in WAS rats by upregulating the stem niche factors WNT3A and TGF$\beta$, which are secreted by Paneth cells (Zhou et al., 2020). Mi et al. (2017) studied the effects of B. infantis in attenuating the severity of chemotherapy-induced intestinal mucositis. They demonstrated that the $B$. infantis group showed higher intestinal villus height and deeper crypt depth than the chemotherapy group. It has been shown that LGG can promote intestinal epithelial homeostasis through specific signaling pathways. Researchers have also found that two proteins secreted from LGG, i.e., p75 (75 Dalton) and P40 (40 Dalton), promote the 
growth of the human and mouse colonic epithelial cells. At the same time, it promotes the growth of mouse colon cells in vitro. The current study screened one B. bifidum FL-228.1 strain that exhibited a higher ability to promote intestinal development than LGG. Overall, the results of this study are consistent with those of previous studies. However, previous studies have only evaluated the function of the intestinal microbiota and the mechanism involved in promoting the development of intestinal epithelial cells. This study was more specific and might be beneficial to the study of probiotic effects in the future. Further research should also focus on specific probiotic functions.

\section{DATA AVAILABILITY STATEMENT}

The datasets presented in this study can be found in online repositories. The names of the repository/repositories and accession number(s) can be found in the article/Supplementary Material.

\section{ETHICS STATEMENT}

All procedures performed in studies involving human participants were in accordance with the ethical standards of the institutional (HRYLL201607). Written informed consent to participate in this study was provided by the participants' legal guardian/next of kin. Written informed consent was obtained

\section{REFERENCES}

Bäckhed, F., Roswall, J., Peng, Y., Feng, Q., Jia, H., Kovatcheva-Datchary, P., et al. (2015). Dynamics and stabilization of the human gut microbiome during the first year of life. Cell Host Microbe 17, 690-703.

Bhinder, G., Allaire, J. M., Garcia, C., Lau, J. T., Chan, J. M., Ryz, N. R., et al. (2017). Milk Fat globule membrane supplementation in formula modulates the neonatal gut microbiome and normalizes intestinal development. Sci. Rep. 7:45274

Blanton, L. V., Charbonneau, M. R., Salih, T., Barratt, M. J., Venkatesh, S., Ilkaveya, O., et al. (2016). Gut bacteria that prevent growth impairments transmitted by microbiota from malnourished children. Science 351:10.1126/science.aad3311 aad3311.

Chong, C. Y. L., Bloomfield, F. H., and O'sullivan, J. M. (2018). Factors affecting gastrointestinal microbiome development in neonates. Nutrients 10:274. doi: 10.3390/nu10030274

Désir-Vigné, A., Haure-Mirande, V., De Coppet, P., Darmaun, D., Le Dréan, G., and Segain, J.-P. (2018). Perinatal supplementation of 4-phenylbutyrate and glutamine attenuates endoplasmic reticulum stress and improves colonic epithelial barrier function in rats born with intrauterine growth restriction. J. Nutr. Biochem. 55, 104-112. doi: 10.1016/j.jnutbio.2017.12.007

Ding, Y. X., Shi, Y., Han, W.-J., and Cui, H. (2016). Regulation of glucocorticoidrelated genes and receptors/regulatory enzyme expression in intrauterine growth restriction filial rats. Life Sci. 150, 61-66. doi: 10.1016/j.lfs.2016.02.079

Friedrich, M. J. (2013). Genomes of microbes inhabiting the body offer clues to human health and disease. JAMA 309, 1447-1449. doi: 10.1001/jama.2013.2824

Hill, C. J., Lynch, D. B., Murphy, K., Ulaszewska, M., Jeffery, I. B., O’Shea, C. A., et al. (2017). Evolution of gut microbiota composition from birth to 24 weeks in the INFANTMET Cohort. Microbiome 5:4.

Jakobsson, H. E., Abrahamsson, T. R., Jenmalm, M. C., Harris, K., Quince, C., Jernberg, C., et al. (2014). Decreased gut microbiota diversity, delayed Bacteroidetes colonisation and reduced Th1 responses in infants delivered by Caesarean section. Gut 63, 559-566. doi: 10.1136/gutjnl-2012-303249 from the individual(s), and minor(s)' legal guardian/next of kin, for the publication of any potentially identifiable images or data included in this article.

\section{AUTHOR CONTRIBUTIONS}

LZL: writing-original draft preparation, formal analysis, and investigation. XZ, MZ, LL, SJ, JP, and YL: samples collections. PG: reviewing and editing. $\mathrm{HN}, \mathrm{JZ}$, and SC: investigation and data curation. XH: project administration. LZ and DC: funding acquisition, supervision, conceptualization, writing-review, and editing. All authors contributed to the article and approved the submitted version.

\section{FUNDING}

This work was financially supported by National Key R\&D Program of China (2018YFC1604302) and the Heilongjiang Province Key Science and Technology Plan (GA16B201-2).

\section{SUPPLEMENTARY MATERIAL}

The Supplementary Material for this article can be found online at: https://www.frontiersin.org/articles/10.3389/fmicb. 2021.626144/full\#supplementary-material

Lau, J. T., Whelan, F. J., Herath, I., Lee, C. H., Collins, S. M., Bercik, P., et al. (2016). Capturing the diversity of the human gut microbiota through culture-enriched molecular profiling. Genome Med. 8:72.

Liu, W., Zhang, J., Wu, C., Cai, S., Huang, W., Chen, J., et al. (2016). Unique features of ethnic mongolian gut microbiome revealed by metagenomic analysis. Sci. Rep. 6:34826.

Lundgren, S. N., Madan, J. C., Emond, J. A., Morrison, H. G., Christensen, B. C., Karagas, M. R., et al. (2018). Maternal diet during pregnancy is related with the infant stool microbiome in a delivery mode-dependent manner. Microbiome 6:109.

Madan, J. C., Hoen, A. G., Lundgren, S. N., Farzan, S. F., Cottingham, K. L., Morrison, H. G., et al. (2016a). Association of cesarean delivery and formula supplementation with the intestinal microbiome of 6-weekold infants. JAMA Pediatr. 170, 212-219. doi: 10.1001/jamapediatrics.2015. 3732

Madan, J. C., Hoen, A. G., Lundgren, S. N., Farzan, S. F., Cottingham, K. L., Morrison, H. G., et al. (2016b). Effects of Cesarean delivery and formula supplementation on the intestinal microbiome of six-week old infants. JAMA Pediatr. 170, 212-219. doi: 10.1001/jamapediatrics.2015.3732

Maldonadolobón, J. A., Gilcampos, M., Maldonado, J., Lópezhuertas, E., Floresrojas, K., Valero, A. D., et al. (2015). Long-term safety of early consumption of Lactobacillus fermentum CECT5716: a 3-year follow-up of a randomized controlled trial. Pharmacol. Res. 95-96, 12-19. doi: 10.1016/j.phrs. 2015.01.006

Martín, R., Jiménez, E., Heilig, H., Fernández, L., Marín, M. L., Zoetendal, E. G., et al. (2009). Isolation of bifidobacteria from breast milk and assessment of the bifidobacterial population by PCR-denaturing gradient gel electrophoresis and quantitative real-time PCR. Appl. Environ. Microbiol. 75, 965-969. doi: 10.1128/aem.02063-08

Meng, Z., Zhang, L., Xin, L., Lin, K., Yi, H., and Han, X. (2018). Technological characterization of Lactobacillus in semihard artisanal goat cheeses from different Mediterranean areas for potential use as nonstarter lactic acid bacteria. J. Dairy Sci. 101, 2887-2896. doi: 10.3168/jds.2017-14003 
Mi, H., Dong, Y., Zhang, B., Wang, H., Peter, C. C. K., Gao, P., et al. (2017). Bifidobacterium Infantis ameliorates chemotherapy-induced intestinal mucositis via regulating T cell immunity in colorectal cancer rats. Cell. Physiol. Biochem. 42, 2330-2341. doi: 10.1159/000480005

Nagpal, R., Tsuji, H., Takahashi, T., Nomoto, K., Kawashima, K., Nagata, S., et al. (2017). Ontogenesis of the gut microbiota composition in healthy, full-term, vaginally born and breast-fed infants over the first 3 years of life: a quantitative bird's-eye view. Front. Microbiol. 8:1388. doi: 10.3389/fmicb.2017.01388

O’Connell Motherway, M., Houston, A., O'callaghan, G., Reunanen, J., O’brien, F., O'driscoll, T., et al. (2019). A Bifidobacterial pilus-associated protein promotes colonic epithelial proliferation. Mol. Microbiol. 111, 287-301. doi: 10.1111/ mmi. 14155

Oh, Y. J., and Jung, D. S. (2015). Evaluation of probiotic properties of Lactobacillus and Pediococcus strains isolated from Omegisool, a traditionally fermented millet alcoholic beverage in Korea. LWT Food Sci. Technol. 63, 437-444. doi: 10.1016/j.lwt.2015.03.005

Pan, W.-H., Li, P.-L., and Liu, Z. (2006). The correlation between surface hydrophobicity and adherence of Bifidobacterium strains from centenarians' faeces. Anaerobe 12, 148-152. doi: 10.1016/j.anaerobe.2006.03.001

Planer, J. D., Peng, Y., Kau, A. L., Blanton, L. V., Ndao, I. M., Tarr, P. I., et al. (2016). Development of the gut microbiota and mucosal IgA responses in twins and gnotobiotic mice. Nature 534, 263-266. doi: 10.1038/ nature 17940

Pragman, A. A., Lyu, T., Baller, J. A., Gould, T. J., Kelly, R. F., Reilly, C. S., et al. (2018). The lung tissue microbiota of mild and moderate chronic obstructive pulmonary disease. Microbiome 6:7.

Reyman, M., Van Houten, M. A., Van Baarle, D., Bosch, A. A. T. M., Man, W. H., Chu, M. L. J. N., et al. (2019). Impact of delivery mode-associated gut microbiota dynamics on health in the first year of life. Nat. Commun. 10:4997.

Riaz Rajoka, M. S., Mehwish, H. M., Siddiq, M., Haobin, Z., Zhu, J., Yan, L., et al. (2017). Identification, characterization, and probiotic potential of Lactobacillus rhamnosus isolated from human milk. LWT Food Sci. Technol. 84, 271-280. doi: 10.1016/j.lwt.2017.05.055

Salam, M. T., Margolis, H. G., Mcconnell, R., Mcgregor, J. A., Avol, E. L., and Gilliland, F. D. (2006). Mode of delivery is associated with asthma and allergy occurrences in children. Ann. Epidemiol. 16, 341-346. doi: 10.1016/j. annepidem.2005.06.054

Shao, Y., Forster, S. C., Tsaliki, E., Vervier, K., Strang, A., Simpson, N., et al. (2019). Stunted microbiota and opportunistic pathogen colonization in caesareansection birth. Nature 574, 117-121. doi: 10.1038/s41586-019-1560-1
Shi, Y.-C., Guo, H., Chen, J., Sun, G., Ren, R.-R., Guo, M.-Z., et al. (2018). Initial meconium microbiome in Chinese neonates delivered naturally or by cesarean section. Sci. Rep. 8, 3255-3255.

Tamburini, S., Shen, N., Wu, H. C., and Clemente, J. C. (2016). The microbiome in early life: implications for health outcomes. Nat. Med. 22, 713-722. doi: $10.1038 / \mathrm{nm} .4142$

Wampach, L., Heintz-Buschart, A., Fritz, J. V., Ramiro-Garcia, J., Habier, J., Herold, M., et al. (2018). Birth mode is associated with earliest strain-conferred gut microbiome functions and immunostimulatory potential. Nat. Commun. 9:5091.

Xu, X., Luo, D., Bao, Y., Liao, X., and Wu, J. (2018). Characterization of diversity and probiotic efficiency of the autochthonous lactic acid bacteria in the fermentation of selected raw fruit and vegetable juices. Front. Microbiol. 9:2539. doi: $10.3389 /$ fmicb.2018.02539

Zhou, C., Fang, X., Xu, J., Gao, J., Zhang, L., Zhao, J., et al. (2020). Bifidobacterium longum alleviates irritable bowel syndrome-related visceral hypersensitivity and microbiota dysbiosis via Paneth cell regulation. Gut Microbes 12:1782156. doi: $10.1080 / 19490976.2020 .1782156$

Conflict of Interest: SJ, JP, and YL were employed by Heilongjiang Feihe Dairy Co., Ltd.

The remaining authors declare that the research was conducted in the absence of any commercial or financial relationships that could be construed as a potential conflict of interest.

Publisher's Note: All claims expressed in this article are solely those of the authors and do not necessarily represent those of their affiliated organizations, or those of the publisher, the editors and the reviewers. Any product that may be evaluated in this article, or claim that may be made by its manufacturer, is not guaranteed or endorsed by the publisher.

Copyright (c) 2021 Lyu, Zhou, Zhang, Liu, Niu, Zhang, Chen, Gong, Jiang, Pan, Li, Han, Cheng and Zhang. This is an open-access article distributed under the terms of the Creative Commons Attribution License (CC BY). The use, distribution or reproduction in other forums is permitted, provided the original author(s) and the copyright owner(s) are credited and that the original publication in this journal is cited, in accordance with accepted academic practice. No use, distribution or reproduction is permitted which does not comply with these terms. 